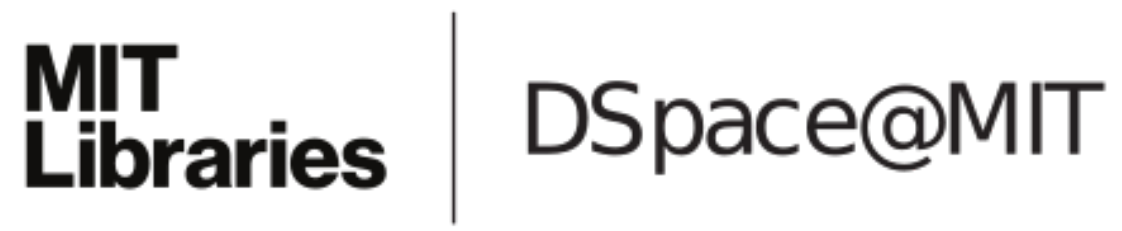

\author{
MIT Open Access Articles
}

The Effect of Stress on Battery-Electrode Capacity

The MIT Faculty has made this article openly available. Please share how this access benefits you. Your story matters.

Citation: Bucci, Giovanna et al. "The Effect of Stress on Battery-Electrode Capacity." Journal of The Electrochemical Society 164, 4 (2017): A645-A654 @ 2017 Electrochemical Society

As Published: http://dx.doi.org/10.1149/2.0371704JES

Publisher: Electrochemical Society

Persistent URL: http://hdl.handle.net/1721.1/120733

Version: Final published version: final published article, as it appeared in a journal, conference proceedings, or other formally published context

Terms of use: Creative Commons Attribution-NonCommercial-NoDerivs License 


\title{
The Effect of Stress on Battery-Electrode Capacity
}

\author{
Giovanna Bucci, ${ }^{\mathrm{a}, \mathrm{z}}$ Tushar Swamy, ${ }^{\text {a }}$ Sean Bishop,,${ }^{\mathrm{a}, *}$ Brian W. Sheldon, ${ }^{\mathrm{b}}$ Yet-Ming Chiang, ${ }^{\mathrm{a}, *}$ \\ and W. Craig Carter ${ }^{a, *}$ \\ ${ }^{a}$ Department of Materials Science and Engineering, Massachusetts Institute of Technology, Cambridge, Massachusetts \\ 02139-4307, USA \\ ${ }^{b}$ School of Engineering, Brown University, Providence, Rhode Island 02912, USA
}

\begin{abstract}
Constraint-induced stresses develop during Li-ion battery cycling, because anode and cathode materials expand and contract as they intercalate or de-intercalate Li. We show in this manuscript that these stresses, in turn, can significantly modify the maximum capacity of the device at a given cell voltage. All-solid-state batteries impose an external elastic constraint on electrode particles, promoting the development of large stresses during cycling. We employ an analytic and a finite element model to study this problem, and we predict that the electrode's capacity decreases with increasing matrix stiffness. In the case of lithiation of a silicon composite electrode, we calculate $64 \%$ of capacity loss for stresses up to $2 \mathrm{GPa}$. According to our analysis, increasing the volume ratio of Si beyond $25-30 \%$ has the effect of decreasing the total capacity, because of the interaction between neighboring particles. The stress-induced voltage shift depends on the chemical expansion of the active material and on the constraint-induced stress. However, even small voltage changes may result in very large capacity shift if the material is characterized by a nearly flat open-circuit potential curve.

(C) The Author(s) 2017. Published by ECS. This is an open access article distributed under the terms of the Creative Commons Attribution Non-Commercial No Derivatives 4.0 License (CC BY-NC-ND, http://creativecommons.org/licenses/by-nc-nd/4.0/), which permits non-commercial reuse, distribution, and reproduction in any medium, provided the original work is not changed in any way and is properly cited. For permission for commercial reuse, please email: oa@electrochem.org. [DOI: 10.1149/2.0371704jes] All rights reserved.

(cC) BY-NC-ND
\end{abstract}

Manuscript submitted September 14, 2016; revised manuscript received January 11, 2017. Published February 2, 2017.

During charge and discharge of lithium-ion batteries, anode and cathode materials expand and contract as they intercalate or deintercalate Li. Graphite, the most commonly used negative electrode material, shows a volume expansion of up to $10 \% .{ }^{1}$ A much larger (up to $300 \%$ ) volume change is observed in high capacity anode materials such as silicon and tin. ${ }^{2,3}$ Even with moderate values of intercalationinduced strain, large stresses can develop within the microstructure, and eventually cause cell degradation. ${ }^{4}$ Much of the prior work where lithiation-induced stresses have been addressed in detail consider individual particles. ${ }^{5-7}$ This particle fracture exposes new surfaces to the electrolyte. This leads to the formation of an additional solid electrolyte interphase, and results in capacity fade especially after cycling. ${ }^{8}$ Most real electrodes consist of multi-particle arrays, where contact stresses can arise if the individual particles are sufficiently closely packed or jammed together. However, these stresses are localized and probably mitigated by the presence of soft polymer binders.

Loss of connectivity to the current collector through damage of the binder and loss of pore volume have been also identified as failure mechanisms. ${ }^{9}$ The entire cell may also change thickness during charge and discharge due to the difference in volume expansion between negative and positive electrodes. Stresses and delamination at the cell level are generated, and can reduce good electrical contact among the cell components.

To date, the stress-related phenomena that are outlined above have been primarily addressed in electrodes with liquid electrolytes. In these systems, the electrolyte does not substantially constrain the volume expansion of the individual active particles. As noted above, some stress buildup at particle contacts is expected to occur, but the overall average stresses in the active materials will be limited. This has been confirmed with in-situ stress measurements on particle-based electrodes in liquid electrolytes, which show overall stress levels that are below $10 \mathrm{MPa}$ both in tension and in compression. ${ }^{10}$

In contrast to these conventional electrodes, all-solid-state batteries (ASSBs; i.e., those using solid-state electrolytes SSE) are made of almost fully dense composite electrodes. Electrode particles are embedded in a solid matrix made of an electrolyte (conducting $\mathrm{Li}$ ions) mixed with an electronically conducting material. In order to establish good contact at the particle interface (and maintain it over

*Electrochemical Society Member.

${ }^{\mathrm{z} E-m a i l: ~ b u c c i @ \text { mit.edu }}$ cycling), low levels of porosity are sought in the electrode processing. Therefore, the chemical expansion of electrode particles is highly constrained by the electrolyte. With stiff ceramic electrolytes, the constraint-induced stresses that develop during cycling can reach values on the order of $1 \mathrm{GPa}$ or higher. As shown in this manuscript, these stresses, in turn, can significantly modify the maximum capacity of the device at a given cell voltage. Even small stress-induced voltage shifts will drastically lower the capacity of materials with a nearly flat intercalation curve. In the case of a silicon composite electrode, we show that lithiation stresses can reach $2 \mathrm{GPa}$ and the associated capacity loss is $64 \%$ of the theoretical value. Although a variety of proposed nano-structured electrodes-for instance nano-particles encapsulated with a carbon shell ${ }^{11-15}$-have been proposed to improve durability of Si electrodes, these solutions have not considered, yet, the trade-off between durability and the capacity loss by constraining the large volume expansion of Si.

The possible advantages of all-solid-state rechargeable lithium-ion batteries have been described elsewhere (refer to ${ }^{16-22}$ for a review of the field). In one common design of ASSBs, the positive and negative electrodes are composites of active electrode-particles embedded in a solid-matrix admixture of ionic and electronic conducting materials. Cathode electrodes are, in most cases, produced and assembled in the lithiated state. During the first discharge, the cathode particles tend to shrink as they de-intercalate $\mathrm{Li}$ (the expansion of $\mathrm{LiCoO}_{2}$ is, for example, an exception). ${ }^{23}$ If the cathode particles remain bonded to the SSE, the particles will be put in tension, as well as the surrounding SSE matrix. The opposite is generally true for the expansion of the anode particles.

The following simple analogy illustrates many of the concepts presented in this manuscript. A dry sponge will expand as it is exposed to water if it is unconstrained. If an unconstrained sponge is exposed to a humid environment (i.e., a $P_{\mathrm{H}_{2} \mathrm{O}}$ ), it will undergo a strain that is an increasing function of humidity until the sponge's water concentration is in chemical equilibrium with its environment. If the sponge is constrained, it will absorb or desorb water until it comes to a combined chemical and elastic equilibrium with its environment. That the value of equilibrium concentration differs when it is put under compression is evident when squeezing a sponge under water. Water in a sponge will tend to flow from regions of compression until it comes to elasto-chemical equilibrium. The capacity of a sponge to absorb water depends on the applied elastic stress. Replacing the sponge with electrode particles and the constraint with a SSE completes the 
analogy, electrode capacity depends on stress. (In solid materials, the ratio of solute composition to stress-free strain is the Vegard rank-2 tensor coefficient. This paper only treats the isotropic case.)

In principle, a battery could be made with partially lithiated $\mathrm{Si}^{24}$ on both sides of a separator (or partially lithiated $\mathrm{Al}$ which can expand by $100 \%) .{ }^{25}$ The battery could be cycled by alternatively compressing and releasing each side, the side placed under compression becomes the positive electrode until enough $\mathrm{Li}^{+}$passes through the separator to establish equal chemo-mechanical potentials on both sides.

The electro-chemo-mechanical literature has mostly focused on the relationship between tensile stress, fracture and battery performance. The role of intercalation-induced stress on the mechanical failure of electrode particles has been treated elsewhere. ${ }^{26-57}$ Here we consider, instead, the impact of stress on the electrode capacity, only based on solution thermodynamics.

The rate of change of the open-circuit potential (OCP) with respect to applied compressive stress has been measured in commercial lithium-ion pouch cells. ${ }^{58}$ In Ref. 58 the authors relate the stresspotential coupling factor to the expansion characteristic of the electrodes, and propose the development of mechanical force sensors based on intercalation materials.

Because stress is a component that determines the driving force for Li diffusion, it must play a role in battery behavior even before it reaches a critical value for fracture or other dissipative phenomena. The present work treats the role of compressive stress in decreasing cell capacity. The phenomenon is general, but we will focus on ASSBs.

For the diffusion of a species $j$, the diffusion potential $\mathcal{M}_{j}$ is used to generalize the driving forces for diffusion when there are multiple work terms associated with the motion of species $j .5^{59,60}$ The driving force for diffusion of species $j$ is $-\nabla \mathcal{M}_{j}{ }^{c}$ The diffusion of species $j$ stops when $\mathcal{M}_{j}$ is uniform. In this paper, we will assume there are three contributions to the diffusion potential: a chemical potential, a stress potential, and an electric potential. For convenience, we combine the chemical and stress potential into a single term $\varpi_{j}$ which we call the chemo-mechanical potential: $\Delta \bar{\varpi}_{j}$ can be measured with an opencircuit electrochemical cell's voltage when only an ion of species $j$ can traverse the separator.

We consider the case of diffusion by vacancy exchange mechanism in an electrode material with constrained moles of sites $c_{\max }$ available per unit of reference volume (i.e., limited Li solubility). Larché and Cahn ${ }^{59}$ introduced the diffusion potential which becomes ${ }^{\mathrm{d}}$

$$
\mathcal{M}(c, \boldsymbol{\sigma})=\bar{\varpi}_{L i}(c, \boldsymbol{\sigma})=R T \log \frac{c \bar{\gamma}}{c_{\max }-c}-\frac{\Omega}{3} \operatorname{Tr} \boldsymbol{\sigma}
$$

under the assumption of constant elastic coefficients and isotropic chemical expansion. Larché and Cahn did not include electrochemical terms and so $\mathcal{M}$ is equivalent to $\varpi$.

In Eq. 1, the stress potential coupling term is composed of the trace of the Cauchy stress ( $\operatorname{Tr} \sigma=\sigma_{i i}$ ) and of the partial molar volume $\Omega$ of $\mathrm{Li}$ in the host lattice ${ }^{\mathrm{e}}$. The symbol $c$ represents the $\mathrm{Li}$ concentration as the number of moles per unit reference (undeformed ${ }^{f}$ ) volume.

\footnotetext{
${ }^{\mathrm{c}}$ If the motion of species $j$ requires the motion of another species $k$, then gradients of $\mathcal{M}_{k}$ appear in the driving force (see Equations 18 and 19 of Reference 61. In the case treated in this paper, $\mathrm{Li}$ is assumed to exchange with vacancies which have a reference diffusion potential of zero.

${ }^{\mathrm{d}}$ Notation:

- in the reminder of the paper we will drop the index when referring to $\varpi_{L i}$

- $\bar{\gamma}$ is defined as the ratio between the activity coefficient of $\mathrm{Li}$ and the activity coefficient of $\mathrm{Li}$ vacancies

${ }^{\mathrm{e}} \mathrm{We}$ refer to the chemical expansion as the change in lattice dimension and therefore volume, that results from changes in chemical composition, and we express it in terms of the Vegard's parameters. Assuming a material with isotropic chemical expansion, the partial molar volume in Eq. 1 can be written in terms of the isotropic Vegard's parameter $\beta$ as $\Omega=3 \frac{\beta}{\rho_{h}}$, with $\rho_{h}$ being the molar volume of the hosting compound. In case of anisotropic chemical expansion the last term in Eq. 1 reads $-(1 / 3) \Omega_{i i} \sigma_{i i}=-\left(1 / \rho_{h}\right) \beta_{i i} \sigma_{i i}$, with $\beta_{11}, \beta_{22}, \beta_{33}$ being the Vegard's parameters in the three principal directions (see page 1059 of Reference 59).

${ }^{\mathrm{f}} \mathrm{We}$ take the reference configuration to be also stress-free.
}

A detailed derivation of the diffusion potential in the context of nonlinear continuum mechanics theory can be found in Ref. 62. The nonlinear formulation has been employed in the finite element analyzes presented in Solubility of lithium in a silicon all-solid battery electrode - Finite element model section.

At equilibrium, the diffusion potential of a species $j, \varpi_{j}+z_{j} \mathcal{F} \mathcal{E}$ is constant where $\mathcal{F}$ is the faraday constant, $\mathcal{E}$ is the voltage difference with respect to some standard, and $z_{j}$ is charge transfer associated with the diffusion of the species $j$. Therefore, the chemo-mechanical potential can be obtained by measuring changes in the open-circuit potential under superimposed stresses. The stress-OCP coupling has been estimated to be $62 \mathrm{mV} / \mathrm{GPa}$ for sputtered amorphous silicon ${ }^{63}$ in thin-film configurations. Stress-OCP coupling in thin-film Si electrodes has been exploited by mechanical energy harvesters. ${ }^{24}$ Bending-induced asymmetric stresses generate chemical-potential difference, driving lithium ion flux from the compressed to the tensed electrode to generate electrical current up to $20 \mu \mathrm{A} \mathrm{cm}{ }^{2}$. In Ref. 64 at $20 \%$ of the initial discharge, the authors measured a variation in the potential of $36 \mathrm{mV}$ and $50 \mathrm{mV}$, due to an applied pressure of $150 \mathrm{MPa}$ and 230 Mpa respectively. Also, Son et al. ${ }^{65}$ report a shift (of about $100 \mathrm{mV}$ ) to a lower potential for the Si-alloying reaction plateau. Such a voltage shift is due to the compressive stress arising from mechanical confinement of Si particles, and this shift is consistent with a reduced equilibrium Li chemo-mechanical potential in the two phases.

Funayama and coauthors designed a four-point bending test to measure stress-potential coupling in symmetric cells consisting of two $\mathrm{Li}_{0.6} \mathrm{CoO}_{2}$ or $\mathrm{Li}_{0.9} \mathrm{Mn}_{2} \mathrm{O}_{4}$ electrodes and the $\mathrm{Li}_{0.29} \mathrm{La}_{0.57} \mathrm{TiO}_{3}$ electrolyte. The measured voltage change was observed to depend linearly on the applied stress, with the ratios $6.1 \times 10^{-12} \mathrm{~V} / \mathrm{Pa}$ for $\mathrm{Li}_{0.6} \mathrm{CoO}_{2}$ and $1.9 \times 10^{-12} \mathrm{~V} / \mathrm{Pa}$ for $\mathrm{Li}_{0.9} \mathrm{Mn}_{2} \mathrm{O}_{4}$-which is a weaker coupling than silicon.

Eq. 1 implies that two different Li concentrations can be in equilibrium if their stress states differ. As qualitatively illustrated in Fig. 2 , the same value of diffusion potential corresponds to a higher concentration in a stress-free material, with respect to a material under compressive stress (in combination with positive Vegard's parameters).

The effect will be accentuated in systems with larger Vegard's parameters, such as $\mathrm{Li}$ in $\mathrm{Si}$. The stress-OCP coupling is limited by the value of stress that the material can withstand without fracturing, or by the characteristic yield stress if the material is ductile. The magnitude of the stress-OCP coupling for a specific electrode material depends on the shape of the intercalation curve (i.e., on the derivative of the potential with respect to Li content). A larger deviation of $\mathrm{Li}$ concentration with stress will occur in systems with a relatively 'flat' open-circuit potential (OCP) curve. Referring to Fig. 2, the capacity shift can be estimated as

$$
\Delta c \approx \frac{\frac{\Omega}{3} \operatorname{Tr} \sigma}{\partial(O C P) / \partial c}
$$

Because $\partial(O C P) / \partial c$ depends on the enthalpy of mixing, the local value of the activity coefficient $\bar{\gamma}(c)$ affects the stress-induced shift in Li solubility. The role of $\bar{\gamma}(c)$ has been investigated in Ref. 66 in the context of a thin-film Si electrode. Compressive stresses reaching $1 \mathrm{GPa}$ have been estimated to alter the electrochemical potential by $50 \mathrm{mV}$. However, the largest stress is attained at a smaller lithiation state where the OCP curve is steep, and the authors calculated a decrease in Li content of about $20 \%$.

The stress-OCP coupling is expected to be larger for electrode materials characterized by large chemical expansions. Silicon is an extreme case, with about $270 \%$ of volumetric expansion with the insertion of 3.75 moles of Li per mole of Si. The large Vegard constant of $\mathrm{Li}$ in $\mathrm{Si}$ is known to diminish the durability of Si composite electrodes. ${ }^{64}$ Concentration gradients can cause shear stresses sufficient to pulverize the Si particles, ultimately causing loss of electrical contact.

In general Vegard's stresses develop in electrode particles depending on the level of mechanical constraints imposed by the surrounding matrix, such as a solid electrolyte, a binder, or an active-particle 

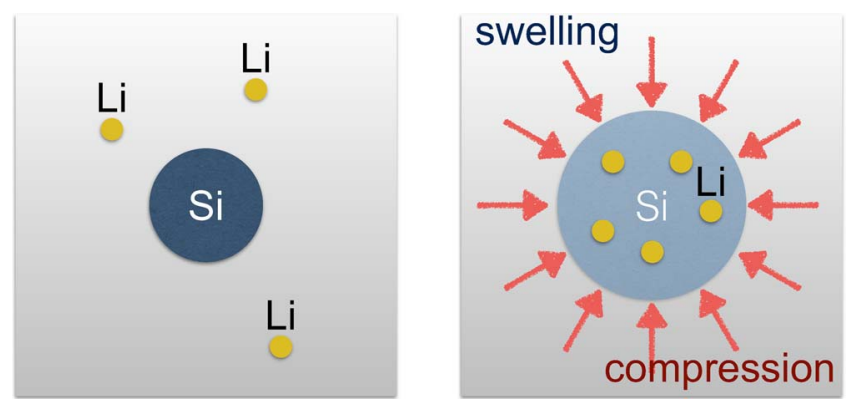

Figure 1. A silicon particle in a solid matrix is modeled in Solubility of lithium in a silicon all-solid battery electrode - Analytical model of a spher ical inclusion section as a spherical inclusion in an infinite matrix. As Li diffuses in the particle, Si chemical expansion is constrained by the surrounding matrix. Compressive stresses will develop within the particle. The magnitude of the particle's compressive stress increases with matrix stiffness.

encapsulating protective-shell (in core-shell topology). Mechanical confinement provided by an electrochemically inactive matrix or coating has been proposed ${ }^{64,65}$ as a viable solution to limit the volume expansion of $\mathrm{Si}$. Such methods show advantages similar to a variety of proposed nano-structured electrodes (for instance nano-particle encapsulation with a carbon shell ${ }^{1-15}$ ).

However, stress-OCP coupling should diminish the capacity in systems where Si particles are not free to expand. Piper et al. ${ }^{64}$ investigated a system of $\mathrm{Si}$ and glassy solid electrolyte $\left(77.5 \mathrm{Li}_{2} \mathrm{~S}-\right.$ $22.5 \mathrm{P}_{2} \mathrm{~S}_{5}$ ), and showed that volume confinement of Si particles limits achievable capacity, and allows for higher capacity retention. The application of an external load (150 and $230 \mathrm{MPa})$ contributes to a larger compressive stress and consequently to a lower Li solubility. Given silicon's large theoretical capacity, confinement creates a compromise between energy density and durability.

Similarly, Son et al. ${ }^{65}$ employed melt spinning to embed nano-Si particles in a $\mathrm{Ti}_{4} \mathrm{Ni}_{4} \mathrm{Si}_{7}$ matrix, which acts as a mixed conductor. In such a constrained microstructure, lithiation is limited to $900 \mathrm{mAhg}^{-1}$ (almost $1 / 4$ of the theoretical capacity), and a coulombic efficiency of $99.7 \%$ is maintained after 50 cycles.

Motivated by the interest in silicon as a high capacity anode material and by the recent experimental evidence for stress-limited lithiation, ${ }^{64,65}$ we analyze the case of $\mathrm{Si}$ anode particles embedded in an infinite solid-electrolyte matrix, taking the electrolyte's Young's modulus as a parameter that we will vary in the model. Analytical solutions for spherical inclusions in an infinite matrix are known. ${ }^{68}$ Using an experimental OCP curve for $\mathrm{Si}$, we calculate the effect of stress on capacity in Solubility of lithium in a silicon all-solid battery electrode - Analytical model of a spher ical inclusion section. We also estimate stress-potential coupling effects for a generic spherical electrode particle as a function of its chemical expansion and its stress state (assuming no fracture or other irreversible elastic effects).

In Solubility of lithium in a silicon all-solid battery electrode Finite element model section, we compare the results from Solubility of lithium in a silicon all-solid battery electrode - Analytical model of a spher ical inclusion section with data from a finite element model (FEM) of a composite microstructure. In the finite element analyses, we model an assemblage of square particles. In addition to investigating the effect of a finite matrix, the FEM calculations reveal the effect of stress concentrations due to jagged interfaces. The stress concentrations are likely sites of fracture initiation and its associated loss of electrical contact. We discuss the conditions for formulating the diffusion potential within a nonlinear continuum mechanics approach (Eq. A1). ${ }^{62}$

\section{Solubility of Lithium in a Silicon All-Solid Battery Electrode - Analytical Model of a Spherical Inclusion}

We consider a $\mathrm{Si}$ electrode particle embedded in a matrix, representing a composite material made of solid electrolyte and an

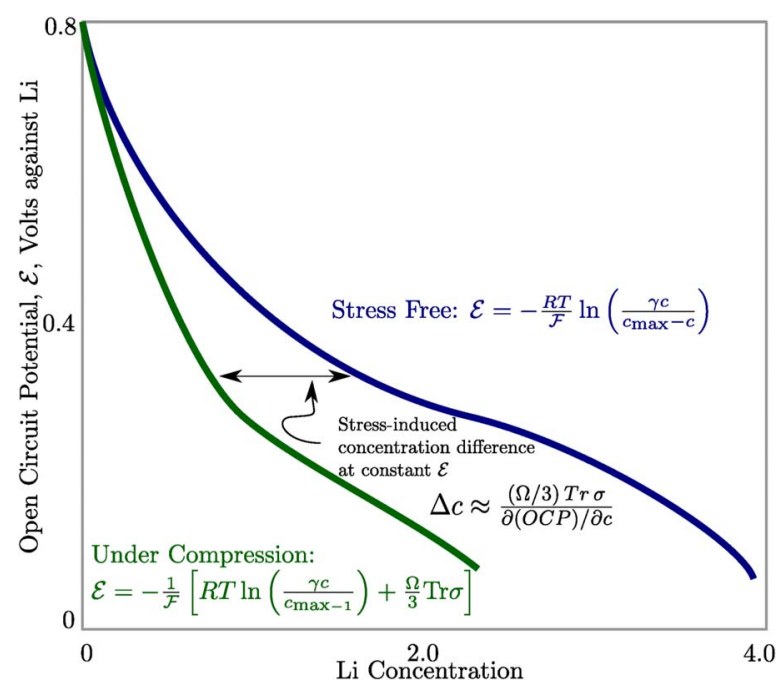

Figure 2. The diffusion potential is a generalization of the chemical potential, in the sense that it includes several driving forces for diffusion, in addition to chemical composition. The figure above highlight the contribution of compressive stress on the total diffusion potential for a material with positive Vegard's parameters. Points characterized by the same diffusion potential (an equilibrium state), have two different values of concentration. The shift in concentration $\Delta c$ depends (according to Eq. 2) on pressure and on the tangent to the open-circuit potential curve (at a certain state of charge). It follows that a larger overpotential is required to intercalate $\mathrm{Li}$ in a particle that is under compressive stress.

electronic-conductive additive (see Fig 1). In this model, the particle's interfacial traction depends on the Young's modulus of the matrix region. We will quantify the effect of such confinement by calculating the maximum capacity that the particle can achieve based on solution thermodynamics. Electrode and electrolyte materials are modeled as isotropic. We assume silicon to be amorphous-crystalline Si turns amorphous by alloying with lithium during the first lithiation cycle-and to behave isotropically.

The hydrostatic pressure $P$ within a spherical inclusion (of Young's modulus $\mathrm{E}_{p}$, Poisson's ratio $\nu_{p}$ and Vegard's parameter $\beta$ ) in an infinite isotropic matrix region (with the modulus of elasticity $\mathrm{E}_{m}$, Poisson's ratio $v_{m}$ and no matrix chemical expansion) is

$$
p=\frac{\beta\left(c / c_{\max }\right)}{\frac{1+v_{m}}{2 E_{m}}+\frac{1-2 v_{p}}{E_{p}}}
$$

Eq. 3 has been used to calculate thermal-expansion-misfit stresses during cooling of a composite material. ${ }^{67}$ According to Eq. 3, the pressure is constant within the particle, and is independent of particle size.

In amorphous silicon thin-film electrodes, the material has been observed to behave elastically up to a biaxial stress of approximately 1 GPa ${ }^{63}$ For a spherical inclusion, the stress state arising from isotropic volumetric expansion is purely hydrostatic. ${ }^{68}$ In absence of a deviatoric component, the von Mises stress is zero and no plastic deformation occurs. For constant elastic properties, Eq. 3 shows a linear dependence of the pressure on Li concentration as illustrated in Fig. 3. The pressure is plotted over a limited range of Li concentration, corresponding to the Li solubility predicted by the model and explained below. Eq. 3 tends to overestimate the pressure by assuming the matrix is infinite. We compare these values with stresses predicted by the finite element model at the end of Solubility of lithium in a silicon all-solid battery electrode - Finite element model section.

The stress-OCP coupling in the the electrode particle is computed according to Eq. 1. The stress-dependent term of Eq. 1 is zero in the matrix region, because the trace of the Cauchy stress (i.e., the pressure) is zero. ${ }^{67}$

To illustrate the effect of matrix stiffness, we choose four different values of the matrix's Young's modulus, $\mathrm{E}_{\text {matrix }}=5,10,15,20 \mathrm{GPa}$. 


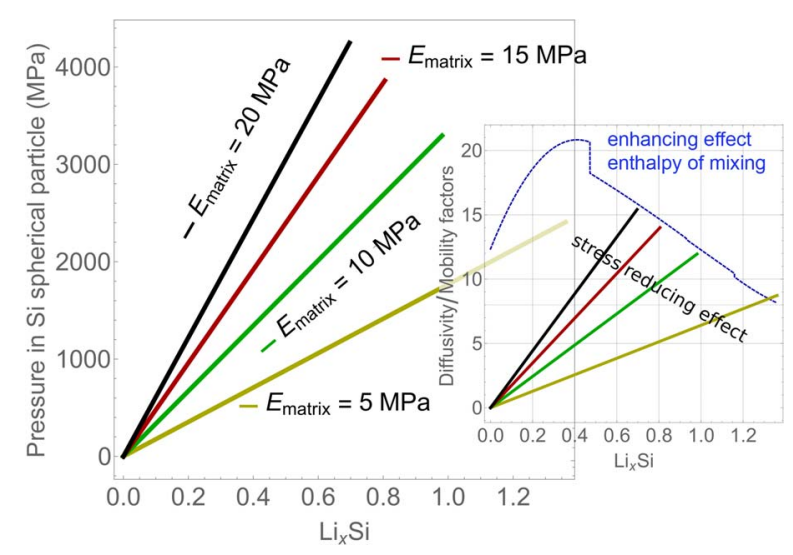

Figure 3. The pressure developing in a spherical inclusion due to chemical expansion depends linearly on the concentration (left image in Fig. 3). The stiffer the surrounding matrix the larger is the pressure in the particle. Compressive stress in the order of $2 \mathrm{GPa}$ also reduces Li chemical diffusivity (described in Impact of hydrostatic compressive of stress-free conditions section) by one order of magnitude. The upper-right plot shows stress-dependent factor which relates the effective chemical diffusivity to Li mobility according to Eq. 6. The stress-dependent reducing factor $\frac{\Omega c}{R T} \frac{\partial p}{\partial c}$ (represented by the four straight lines in the right plot) has a negative sign. The enthalpy of mixing of $\mathrm{Li}$ in $\mathrm{Si}$ tends to enhance diffusivity, instead. The corresponding thermodynamic factor $\left(\frac{c_{\max }}{\left(c_{\max }-c\right)}+\frac{\partial \log \bar{\gamma}}{\partial \log c}\right)$ in Eq. 6 is represented by the blue dashed curve.

The higher end of this range of values is realistic for sulfide solid electrolytes. The Young's modulus of $\mathrm{Li}_{2} \mathrm{~S}-\mathrm{P}_{2} \mathrm{~S}_{5}$ sulfide solid electrolytes has been estimated (by ultrasonic velocity measurements) to be in the range of 18-25 GPa, for hot pressed pellets, and 14-17 GPa for pellets pressed at room temperature. ${ }^{69}$ Such a low stiffness makes sulfide SSEs an attractive material for the design of bulk-type batteries ${ }^{70}$. We identify a strong relationship between the SSE elastic properties and the capacity extracted from the intercalation material (see Fig. 4). As highlighted in Fig. 4, the solubility of $\mathrm{Li}$ is reduced even for compliant SSEs. An stiffer solid electrolyte would oppose the particles' expansion and reduce Li solubility to impractical values (see Fig. 5). This would be for instance the case of some promising oxide SSEs, such as LLZO $\left(\mathrm{E} \sim 150 \mathrm{GPa}^{71}\right)$ and LLTO $\left(\mathrm{E} \sim 200 \mathrm{GPa}^{72}\right)$.

Solution thermodynamic parameters such as the free energy of mixing for $\mathrm{Li} / \mathrm{Li}_{x} \mathrm{Si}$ can be extracted from the potential vs. composition curves available in literature from experimental measurements ${ }^{74}$ and ab-initio DFT calculations. ${ }^{73}$ The open-circuit potential curve for silicon shows asymmetry between charging and discharging. A characteristic hysteresis has been reported, but without agreement on its cause. ${ }^{75}$ For this study, we consider the OCP curve predicted by DFT calculations in Ref. 73 as the stress-free intercalation curve (blue curve in Fig. 4). This curve appears to be intermediate with respect to observed charge-discharge curves (dotted curves in Fig. 4) ${ }^{73,74}$. Following the procedure described in Ref. 76 solution thermodynamic parameters are determined for the stress-free state. From the stressfree intercalation curve (blue line in Fig. 4), we derive the three curves plotted in Fig. 4 by adding the stress-OCP coupling term. Fig. 4 illustrate the results for the three cases: $\mathrm{E}_{\text {matrix }}=5,10,20 \mathrm{GPa}$. Table II summarizes the reduced capacity predicted by both models in the four cases considered here.

Compressive stress lowers Li solubility for an electrode material with positive Vegard's parameters. Capacity decreases with increasing matrix stiffness. Only $36 \%$ of the theoretical capacity is available in the example with the most compliant solid electrolyte ( $5 \mathrm{GPa})$. In the other cases the capacity reduces from $26 \%$, to $19 \%$ as the stiffness of the solid electrolyte matrix varies from $10 \mathrm{GPa}$ to $20 \mathrm{GPa}$. This result is sensitive to the particular choice of the silicon stress-free intercalation curve. Assuming the experimentally-measured lithiation curve (dotted curve in Fig. 4), the stress-potential coupling effect would generate a reduction in Li solubility of the order of $70-80 \%$. Because stress plays a role in the hysteresis of Si cycling curves, we

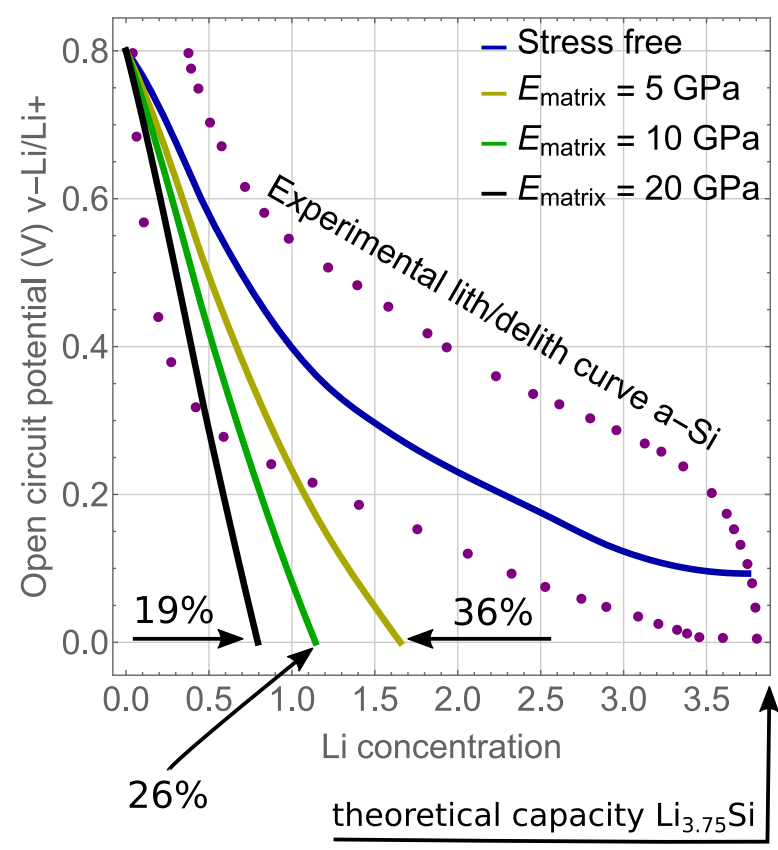

Figure 4. The blue curve identifies the $\mathrm{Si}$ open-circuit potential (OCP) vs. $\mathrm{Li}$, as calculated in Ref. 73. Experimental OCP curves differ in charge and discharge (dotted lines). Increasing compressive stress has the effect of shifting the potential more and more toward the Li reference value, and decreases the storage capacity.

consider the intermediate blue curve of Fig. 4 (confirmed by ab-initio analyses) to be more representative of stress-free conditions.

Impact of hydrostatic compressive stress on diffusivity.-We quantify here the contribution of Vegard's compressive stress on the chemo-mechanical diffusivity (related to the chemical diffusivity ${ }^{60}$ ). Chemo-mechanical diffusivity is interpreted as the factor coupling $\mathrm{Li}$ flux and its concentration gradient according to Fick's first law.

We define mobility the coefficient $M$ relating the flux to the chemomechanical potential gradient as follows ${ }^{60,62}$

$$
\mathbf{J}_{L i}=-\frac{M}{R T} c \frac{\partial \varpi}{\partial \mathbf{x}}
$$

The expression above defines the Li flux in the deformed configuration. It includes the gradient of the diffusion potential $\varpi$ (also defined

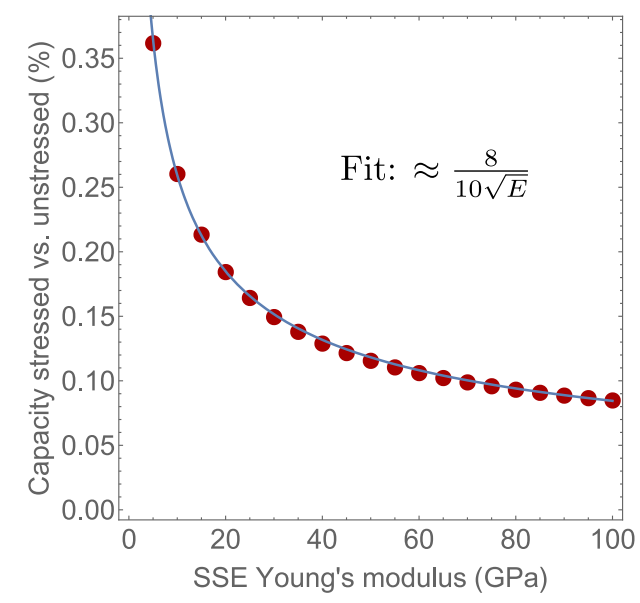

Figure 5. Due to intercalation-induced stresses, the effective capacity of a Si electrode particle depends on the stiffness of the SSE matrix. Even with a relatively compliant sulfide electrolyte (Young's modulus in the range 15-20 GPa) only $20-30 \%$ of the theoretical capacity can be accessed. With typical oxide SSEs, only $10 \%$ of Si storage capacity can be utilized. 
Table I. Material parameters for problems in Solubility of lithium in a silicon all-solid battery electrode - Finite element model section.

\begin{tabular}{|c|c|}
\hline Input value & Description \\
\hline$F=96485.3365 \mathrm{C} \mathrm{mol}^{-1}$ & Faraday's constant \\
\hline$R=8.314 \mathrm{~J} \mathrm{~K}^{-1} \mathrm{~mol}^{-1}$ & gas constant \\
\hline$T=298 \mathrm{~K}$ & temperature \\
\hline$M_{E l}=10^{-10} \mathrm{~m}^{2} \mathrm{~s}^{-1}$ & $\begin{array}{l}\text { mobility of } \mathrm{Li} \text { in the electrode } \\
\text { material }\end{array}$ \\
\hline$M_{S S E}=10^{-10} \mathrm{~m}^{2} \mathrm{~s}^{-1}$ & $\begin{array}{l}\text { mobility of Li in the solid electrolyte } \\
\text { material }\end{array}$ \\
\hline$\rho_{S i}=7.84 \cdot 10^{4} \mathrm{~mol} \mathrm{~m}^{-3}$ & molar density of the hosting material \\
\hline$\rho_{S S E}=208 \mathrm{~mol} \mathrm{~m}^{-3}$ & molar density of the electrolyte \\
\hline$c_{\max _{S i}}=3.75 \times \rho_{S i}$ & $\begin{array}{l}\text { maximum relative number of mole of } \\
\text { Li per mole of electrode compound }\end{array}$ \\
\hline$c_{\max _{S S E}}=0.15 \times \rho_{S S E}$ & $\begin{array}{l}\text { maximum relative number of mole of } \\
\text { Li per mole of solid electrolyte }\end{array}$ \\
\hline$i=10 \mathrm{~A} \mathrm{~m}^{2}$ & $\begin{array}{l}\text { maximum relative number of mole of } \\
\text { Li per mole of solid electrolyte }\end{array}$ \\
\hline$v_{S i}=0.22$ & $\begin{array}{l}\text { Poisson's ratio for Si electrode } \\
\text { particles }\end{array}$ \\
\hline$v_{S S E}=0$ & $\begin{array}{l}\text { Poisson's ratio for solid electrolyte } \\
\text { material }\end{array}$ \\
\hline$\beta_{S i}=0.24$ & $\begin{array}{l}\text { relative lattice constant for } \mathrm{Li} \text { in } \mathrm{Si} \\
\text { electrode material (equivalent to } \\
\Omega=9 \mathrm{~cm}^{3} / \mathrm{mol}\end{array}$ \\
\hline$\beta_{S S E}=0$ & $\begin{array}{l}\text { relative lattice constant for } \mathrm{Li} \text { in the } \\
\text { solid electrolyte material }\end{array}$ \\
\hline$E_{E l}=100 \mathrm{GPa}$ & $\begin{array}{l}\text { Young's modulus of the electrode } \\
\text { material }\end{array}$ \\
\hline$E_{S S E}=5 / 10 / 15 / 20 \mathrm{GPa}$ & $\begin{array}{l}\text { Young's modulus of the solid } \\
\text { electrolyte material }\end{array}$ \\
\hline
\end{tabular}

in the deformed configuration according to Eq. 1), the gas constant $R$, and the absolute temperature $T$. It is convenient to express the chemomechanical potential gradient in terms of the concentration gradient, and determine the thermodynamic factor that relates the mobility $M$ to the chemo-mechanical diffusivity $D$. We present a calculation based on the stress predicted by the spherical inclusion model introduced in this section ${ }^{g}$.

Given the diffusion potential in Eq. 1 as the total driving force for Li diffusion, Li flux can be stated as follows ${ }^{\mathrm{h}}$

$$
\begin{gathered}
\mathbf{J}=-M c\left(\frac{c_{\max }}{c\left(c_{\max }-c\right)}+\frac{\partial \log \bar{\gamma}}{\partial \log c} \frac{1}{c}-\frac{\Omega}{R T} \frac{\partial p}{\partial c}\right) \frac{\partial c}{\partial \mathbf{x}} \\
D=M\left(\frac{c_{\max }}{\left(c_{\max }-c\right)}+\frac{\partial \log \bar{\gamma}}{\partial \log c}-\frac{\Omega c}{R T} \frac{\partial p}{\partial c}\right)
\end{gathered}
$$

We analyze the relative weight of the terms in Eq. 6. Under the assumption of a material with positive chemical expansion, the pressure $p$ has the effect of reducing the diffusivity. Conversely, the thermodynamic factor $b=\left(\frac{c_{\max }}{\left(c_{\max }-c\right)}+\frac{\partial \log \bar{\gamma}}{\partial \log c}\right)$, enhances Li transport. The factor $b$ includes the effect of deviation from ideal solid solution conditions (enthalpy of mixing), and its specific value derives from the intercalation curve adopted here for silicon. In Fig. 3 (top right), we plot the value of the pressure-dependent term $\frac{\Omega c}{R T} \frac{\partial p}{\partial c}$ as a function of the matrix stiffness and Li concentration. In the top-right plot of Fig 3 the value of the factor $b$ is represented by the blue dashed curve. The overall negative contribution of pressure on Li diffusion is counterbalanced by the enhancing effect due to the enthalpy of mixing.

${ }^{\mathrm{g}}$ The mobility $M$ is itself function of pressure and temperature. ${ }^{77}$ However, calculating the effect of pressure on the activation energy for Li transport is beyond the scope of this paper. The interested reader can find analyses and applications in the literature for solid oxide fuel cells. ${ }^{78}$

${ }^{\mathrm{h}}$ In a stress-free material the relationship between diffusivity and mobility is typically expressed by the Darken coefficient $\left(1+\frac{\partial \log \gamma}{\partial \log c}\right)^{60}$. Given the definition of $\bar{\gamma}$ (clarified in footnote 3$)$ the Darken coefficient is replaced by $\left(\frac{c_{\max }}{\left(c_{\max }-c\right)}+\frac{\partial \log \bar{\gamma}}{\partial \log c}\right)$.

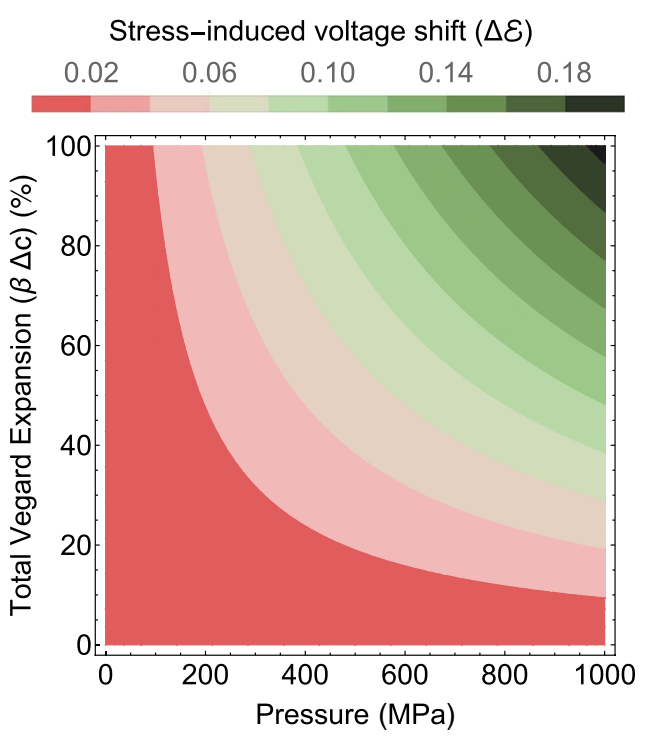

Figure 6. Contour plot showing changes in open-circuit potential caused by stress as a function of the material's chemical expansion and of its stress state. The volume expansion is related to the Li content (and the state of charge) by means of the Vegard's parameters or, equivalently, through the partial molar volume. The pressure can be caused by a constrained chemical expansion or due to an external mechanical load (or a combination of both). The gap between the blue and the green curves of Fig. 2 increases approximately with the state of charge. For a material with positive Vegard's parameters, the volume expansion increases with lithium content. If the material is constrained, compressive stresses arise proportionally to the stress-free expansion.

\section{The General Case - Estimate of Stress-Potential Coupling for Electrode Materials}

The example of silicon treated above is an extreme case. For other materials, we can estimate the values of chemical expansion coefficients at which chemo-mechanical coupling becomes important.

Results are summarized in the contour plot in Fig. 6. The stressinduced potential-shift is computed according to Eq. 1, and the values indicated for the contour line are the differences in voltage with respect to the stress-free state. We consider two variables:

- the material chemical expansion $3 \beta=\rho_{h} \Omega$ (in the form of maximum volume expansion achieved within a given range of stoichiometric variation)

- the pressure $p$ (either externally applied or self-generated by the volume change)

For the input parameters, we made the following assumptions, consistent with realistic conditions discussed above

- positive, isotropic chemical expansion up to $100 \%$ of the initial volume

- compressive hydrostatic stress up to $1 \mathrm{GPa}$

These data cover a parameter range of interest for most electrode materials (experimentally measured Vegard's strains for many Listorage compounds are summarized in Tables 1.1 and 1.2 of Ref. 79, and in Table I of Ref. 23). We calculate a maximum voltage shift of 0.2 Volts under these conditions. Relatively small changes in cell voltage may result in very large stress-induced capacity shift, if the material is characterized by a nearly flat OCP curve. The capacity change with stress in a single-phase system is proportional to $1 /(\mathrm{dV} / \mathrm{dC})$. For two phase systems, such as lithium iron phosphate, the OCP curve has a flat region in which two phases have the same electrochemical potential. In these two-phase regions, the capacity is determined by the miscibility gap: during cycling the phase fractions change as a function of the state of charge. It may seem that, because $1 /(\partial(O C P) / \partial c)$ goes to infinity, the capacity change would be singular. This is not the case. A thermodynamic analysis shows that the capacity is proportional 


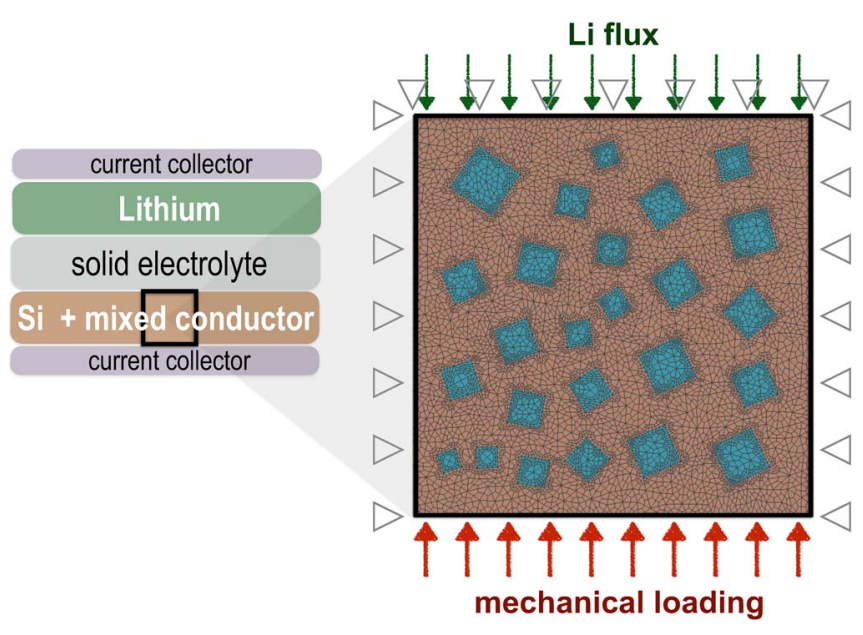

Figure 7. Geometry, discretization and boundary conditions of a finite element model of a composite electrode. Silicon particles are the active material (blue region) embedded in a mixed conductor (brown region) consisting of a solid electrolyte and an electronically conductive additive.

to the $1 /(\partial(O C P) / \partial c)$ values of each single phase evaluated at the limiting miscibility concentrations; these terms are finite for a firstorder phase transition. However, the voltage of the two-phase region and the limiting compositions are a function of stress.

Under the effect of chemical expansion and stress the intercalation curve for an electrode-material (blue curve in the left image of Fig. 6) is shifted by the amount $\Delta \mathcal{E}$ (producing the green curve). The gap between the blue and the green curve tends to increase with the state of charge. For a material with positive Vegard's parameters, the volume expansion increases with lithium content. An applied external load also contributes to the stress-induced potential shift.

\section{Solubility of Lithium in a Silicon All-Solid Battery Electrode - Finite Element Model}

A representative microstructural arrangement of a distribution of particle sizes and its finite element (FE) discretization with linear quadrilateral elements are represented in Fig. 7. In the set of analyses discussed here, the volume ratio of active material is set to about $25 \%$. Such a low $\mathrm{Si}$ volume ratio allows us to compare results from the finite element analyses with the model illustrated in Solubility of lithium in a silicon all-solid battery electrode - Analytical model of a spher ical inclusion section. The spherical inclusion model is independent of the particle's size, and does not include any interaction between neighboring particles. The mechanical interaction between neighboring particles depends on the relative content of silicon in the microstructure. The stress field generated by all the particles expanding collectively grows higher as the particles become closer. Also, in Ref. 64, the authors designed the composite Si electrode with 1:1:5 weight ratio of crystalline Si powder, copper powder and glass solid electrolyte (77.5 $\left.\mathrm{Li}_{2} \mathrm{~S}-22.5 \mathrm{P}_{2} \mathrm{~S}_{5}\right)$; this corresponds to a 1:6 volume ratio of active material to $\mathrm{SSE} /$ carbon matrix. The role of particle interaction in a dense microstructure is discussed further below.

The finite element mesh is representative of a portion of the composite electrode (square highlighted in Fig. 7). The microstructure includes randomly oriented square particles in a region of dimensions $11 \mu \mathrm{m} \times 11 \mu \mathrm{m}$. The average particle size is $1 \mu \mathrm{m}$. The particles position and size distribution follow from a centroidal Voronoi tessellation generated from random seeds.

We consider shapes with sharp corners (like the squares chosen here) to be a more realistic representation of particles than circles (see for instance Fig. 6 in Ref. 70). Flaws and stresses are more likely to accumulate around sharp corners and therefore corners are likely be associated with stress concentration and onset of plastic deformation and/or damage.

\begin{abstract}
Table II. Predicted capacity extracted from the electrode particles (as percentage with respect to the theoretical value) in relation to the stiffness of the solid electrolyte matrix. The comparison between values computed by mean of a spherical inclusion model and the finite element model shows very good agreement.
\end{abstract}

\begin{tabular}{lll}
$\begin{array}{l}\text { Limit Capacity } \\
\text { Analytic }\end{array}$ & FEM & matrix Young's modulus \\
\hline $36 \%$ & $30 \%$ & $5 \mathrm{GPa}$ \\
$26 \%$ & $26 \%$ & $10 \mathrm{GPa}$ \\
$21 \%$ & $24 \%$ & $15 \mathrm{GPa}$ \\
$19 \%$ & $22 \%$ & $20 \mathrm{GPa}$ \\
& $19 \%$ & $20 \mathrm{GPa}$, displacement BC
\end{tabular}

The average particle size does not significantly affect the results, as long as the volume ratio of active material is kept constant and the system is in equilibrium. The stress field is sensitive to particle size when the charging rate is high enough (or Li mobility is low enough) that a diffusion potential gradient arises within the particles and through the electrode. We compute the maximum capacities by assuming that the electrode is charged quasi-statically

Analyses are carried out by means of an in-house FE code implemented according the theoretical continuum model described in full details in Ref. 62. The electrochemical-mechanical problem to be solved at each iteration of the Newton-Raphson algorithm consists of three coupled equations with the unknowns: displacements, Li concentration and diffusion potential. ${ }^{i}$ The constitutive behavior for the electrode and the electrolyte material is assumed to be elastically and diffusively isotropic. No viscoplastic or damage phenomena are taken into account. The input parameters for this problem are summarized in Table. II.

Galvanostatic tests are performed by applying a constant uniform lithium flux (corresponding to a constant current density) at the interface in contact with the solid electrolyte (top edge in Fig. 7). A zero flux is assumed on the remaining edges as Neumann boundary conditions for the diffusion problem. For the mechanical problem, Dirichlet boundary conditions are applied on the left and right boundaries by setting the displacements equal to zero. The displacement of the top edge is considered fully constrained by the presence of the solid electrolyte. At the bottom edge, three different values (3/150/230 MPa) of external pressure are held constant during the operation in order to simulate the conditions in Ref. 64. We observe that varying the mechanical load does not appreciably affect the results. On the contrary, switching from boundary conditions of constant pressure (in the range 3-230 MPa) to a perfectly constrained interface produces an increase in the particles' stress level (compare dashed to continuous black line in Fig. 8).

The compressive stresses computed from the FE model and the approximate analytic calculation can be observed in Fig. 8. The models can be compared via the thick and thin lines of the same shade (representing elastic modulus). The spherical-inclusion model assumes linear dependence of the pressure on Li content over the entire stoichiometric range. The FE model predicts the same linear behavior at low concentration values, but with a higher slope. As the state of charge increases, the incremental pressure with Li content becomes smaller. For concentration values close to the solubility limit, the analytic and numerical models predict very similar values of pressure. The evolution of Si elastic properties with Li concentration does not appreciably affect the results in our analyses. Therefore, we present only data produced under the assumption of constant elastic properties.

To our knowledge the only set of experiments with conditions comparable to the system modeled here are the ones reported by Ref. 64. The large decrease in capacity predicted by our models is in

${ }^{\mathrm{i}}$ With respect to the finite element model described in the supplementary material of Ref. 62 , we take the third unknown to be the entire diffusion potential and not just the stressdependent term. This is a more appropriate choice to model diffusion across materials with different Li solubility. 


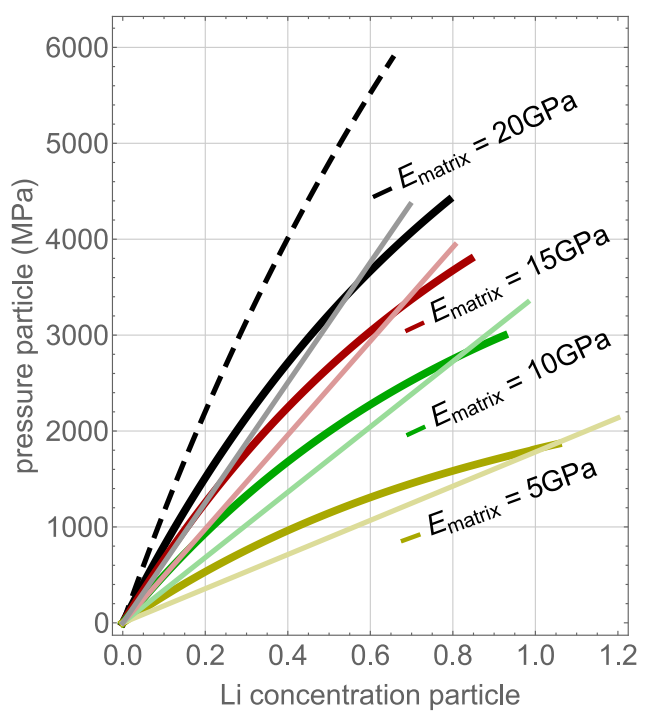

Figure 8. Hydrostatic compressive Cauchy stress predicted by the finite element model is plotted (in thick solid lines) in comparison with the pressure (lighter lines in the same colors) predicted by the analytic model discussed in Solubility of lithium in a silicon all-solid battery electrode - Analytical model of a spher ical inclusion section. We consider the average stress value inside the Si particles and away from corners (points of stress localization). No appreciable difference has been found in the values predicted inside different particles. The dashed black curve refers to the case of $\mathrm{E}_{\text {matrix }}=20 \mathrm{GPa}$, with a zero displacement condition imposed on all the boundary (see Fig. 7).

qualitative agreement with the low capacity of Si electrodes measured by Ref. 64. However, a direct comparison of the results is not possible, because the initial density and stiffness of the solid-electrolyte region are unknown for the samples of Ref. 64. In Ref. 64, the pressure applied to the composite electrodes during assembly is only 5 ton/meter (about $5 \times 10^{-2} \mathrm{MPa}$ ). This assembly pressure is much lower than the molding pressure of $360 \mathrm{MPa}$ reported in Ref. 69,70 to achieve dense sulfide-glass electrolyte pellets at room temperature with Young's moduli of about $20 \mathrm{GPa}$. By reducing the molding pressure to $180 \mathrm{MPa}$, Sakuda et al. ${ }^{69}$ observed the Young's modulus had dropped to $8.8 \mathrm{GPa}$. We expect the mechanical loading of $150 / 230$
MPa maintained during cell operation in Ref. 64 to have the effect of increasing the solid electrolyte density. We do not observe any affect of the applied external pressure in our simulations, because we assume a fully dense composite electrode from the beginning of the analyses. Piper et al. measured a first cycle capacity of $42 \%$ of the theoretical value when $230 \mathrm{MPa}$ of pressure was applied to the cell. As our analyses show, a more severe reduction of Li solubility should be expected if the composite electrode is molded at higher pressure before cycling.

The presence of particles with sharp corners introduces the possibility of a deviatoric component of the stress to arise, in particular at the particle corners. An extension of the constitutive model to include a viscoplastic behavior would bound the stress value to its yield limit and allow for larger capacity extraction. However, further knowledge of the constitutive behavior of sulfide solid electrolyte is required to calibrate the model and make it predictive.

The stress-potential coupling is also located at the value of stress that the material can withstand without fracturing. For a system mostly under compressive stress, we expect fracture to be limited at the particle corners, where tensile stress may arise from particles misalignment. Crack formation allows for stress release in the proximity of the crack tip.

Finally, we analyzed the effect of active material loading on the total available capacity. A more densely packed microstructure further decreases the capacity per unit volume of silicon. Compressive stress grows quickly as the SSE buffer zone between the particles becomes thinner. The spherical inclusion problem discussed earlier is based on the hypothesis of infinitely diluted inclusions. The elastic field due to a spherical inclusion embedded within a spherical representative volume has been studied in Ref. 80. Li et al. calculated the Eshelby tensor under the assumption of homogeneous Dirichelet boundary conditions on the representative volume (i.e. zero displacements applied to the external boundary $)^{80}$. For an isotropic expansion of the inclusion, this solution relates to the Eshelby tensor for the infinite domain ${ }^{81}$ by mean of the factor $\left(1-(r / R)^{3}\right)$, where $r$ and $R$ are the radii of the inclusion and of the surrounding volume, respectively. This factor represents the effect of the boundary when the ratio $r / R$ is non-negligible. The stress cannot be alleviated by reducing particle size, if the volume ratio of active material is kept constant.

In Fig. 9 we illustrate how the average value of $r / R$ can be extracted from the construction of the microstructure based on a centroidal Voronoi tessellation. The plot in the center of Fig. 9 represents the decrease in capacity per unit of $\mathrm{Si}$ volume (expressed in percentage
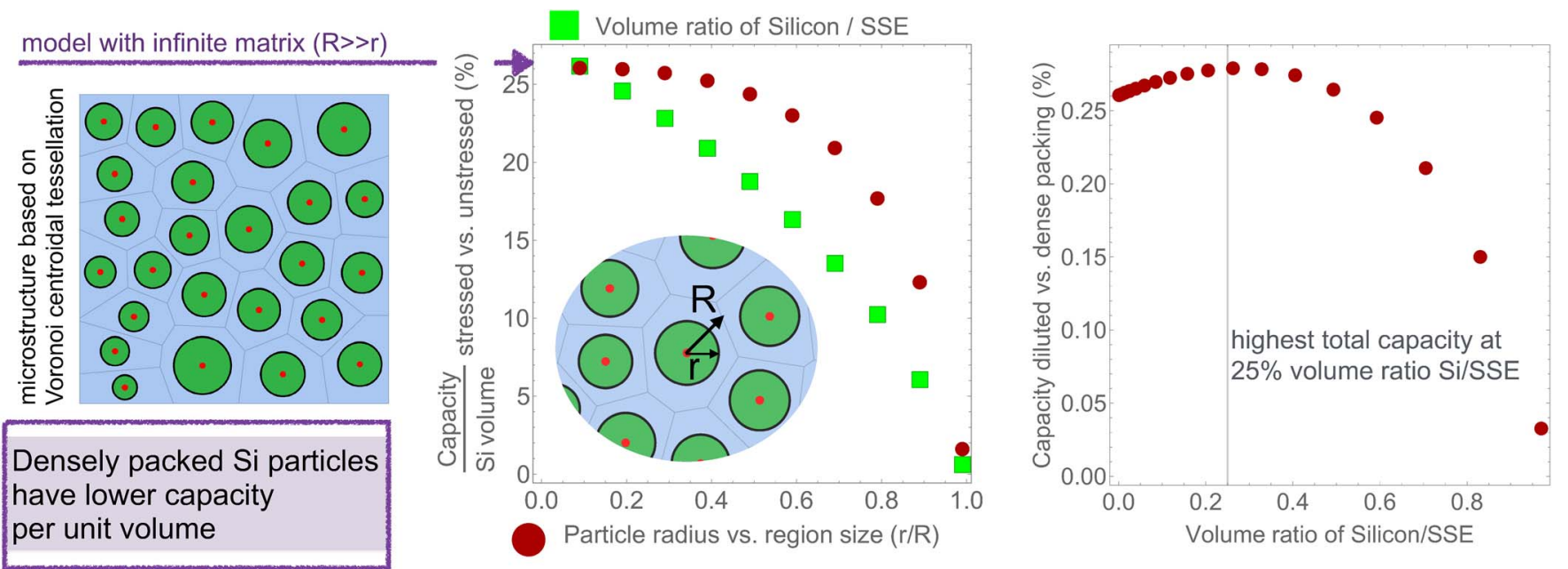

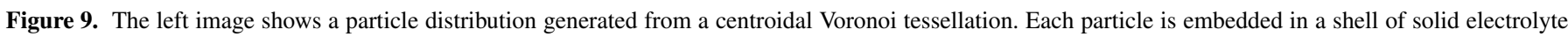

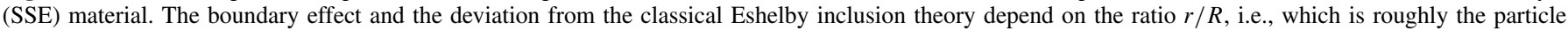

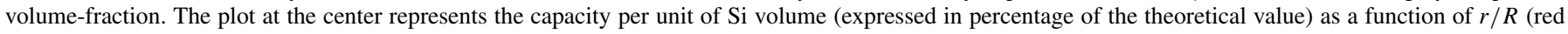

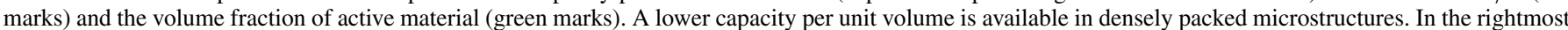

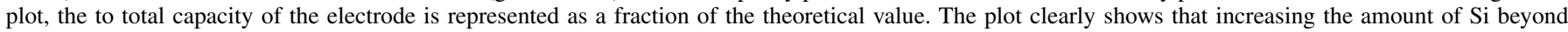
$25-30 \%$ in volume ration is counterproductive. The total capacity will decrease due to large compressive stresses caused by silicon's expansion. 
of the theoretical value for $\mathrm{Li}_{3.75} \mathrm{Si}$ ) as the distance between particles decreases. The level of packing is represented by the ratio $r / R$ (bottom horizontal axis and red marks) and by the volume ratio of active material (green marks). The data plotted in Fig. 9 refer to the case of solid electrolyte having a Young's modulus of $10 \mathrm{GPa}$. In the limit of $r / R \rightarrow 0$, the result of $26 \%$ is recovered, showing consistency with the infinitely dilute model of Solubility of lithium in a silicon all-solid battery electrode - Analytical model of a spher ical inclusion section. In the finite element model, we assume a $25 \%$ of $\mathrm{Si}$ volume ratio. This volume ratio is an average. The ratio varies spatially. Such a low volume ratio implies a drop of only $3-4 \%$ in capacity per unit of $\mathrm{Si}$ volume, caused by neighboring effects.

The rightmost plot in Fig. 9 shows the total storage capacity as a function of the volume ratio of active material. The optimal Si loading appears to be around $25 \%$. Increasing the content of silicon beyond 25-30\% becomes counterproductive, as the stress in a densely packed microstructure reduces the total effective capacity. At the optimal 25\% loading, the total capacity is only slightly larger than the capacity in the limit of a dilute system. This near-equivalence is consistent with the agreement of the FEM calculation and the spherical-inclusion model.

\section{Conclusions}

Compressive stresses reduce cell capacity. The amount of capacity loss can be quantified through a simple model which is in agreement with a finite element model of a battery electrode microstructure. We have showed an example of intercalation-induced compressive stress limiting the capacity of a Si electrode to $20 \%$ of its theoretical value. Solid-state electrolytes (SSE) provide an elastic constraint to electrode particles and constraint-induced stresses develop during cycling. The focus of our study was for all-solid-state batteries, however the result is applicable to any electrochemical system where stresses arise or are applied. We analyze the case of Si anode particles embedded in a solid-electrolyte matrix, taking the electrolyte's Young's modulus as a variable of the problem. Both analytic and a finite element model predict that storage capacity decreases with increasing matrix stiffness. According to the spherical-inclusion model discussed in Solubility of lithium in a silicon all-solid battery electrode - Analytical model of a spher ical inclusion section, only $36 \%$ of the theoretical capacity is available in the example with the most compliant solid electrolyte (5 $\mathrm{GPa})$. In the other cases the capacity reduces from $26 \%$, to $19 \%$ as the stiffness of the solid electrolyte matrix varies from $10 \mathrm{GPa}$ to $20 \mathrm{GPa}$. This range of elastic stiffness is realistic for sulfide SSEs. Less compliant solid electrolyte materials would further limit Si lithiation. Higher loading fractions of Si particles do not necessarily lead to higher energy density. This surprising conclusion underscores the importance of accounting for electro-chemo-mechanical coupling. We determine the optimal volume ratio of silicon to be around $25 \%$, as this leads to the highest total capacity. Given Si large theoretical capacity, this value is still of practical interest as it also leads to improved reliability.

The stress-induced voltage shift is accentuated in systems with large chemical expansion, such as $\mathrm{Li}$ in $\mathrm{Si}$. However, even a small change in the cell voltage may result in a large capacity shift if the active material is characterized by a nearly flat open-circuit potential curve.

\section{Acknowledgments}

The work was supported by the grant DE-SC0002633 funded by the U.S. Department of Energy, Office of Science. BWS also acknowledges support by the US Department of Energy, Office of Basic Energy Sciences under Contract DE-FG02-10ER46771.

\section{Appendix A. Stress-Potential Coupling in Linear vs Nonlinear Kinematics}

The stress-dependent component of the diffusion potential derived in Ref. 62 has the following form

$$
\varpi^{M}\left(c, \mathbf{C}^{e}\right)=-\frac{\Omega}{3} \operatorname{Tr}\left(\mathbf{F}^{e T} \mathbf{P}\right)
$$

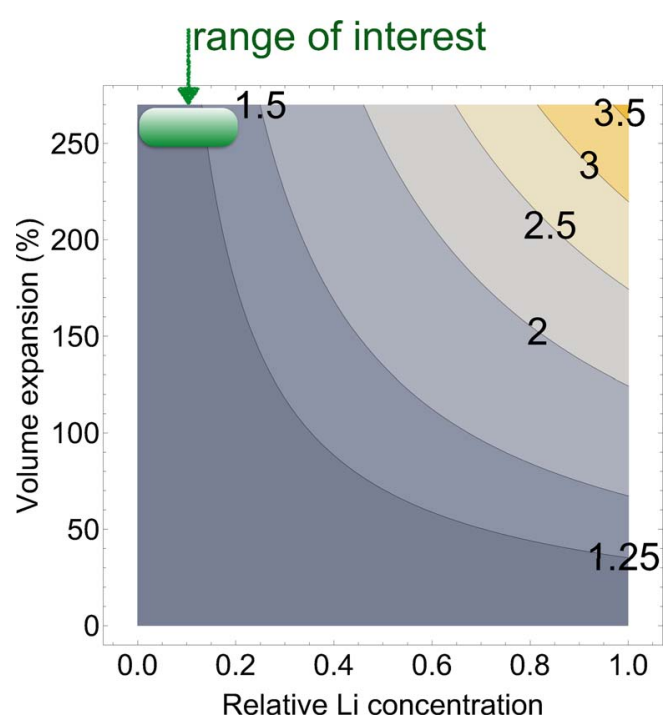

Figure 10. Correction factor between the linear approximation of stress overpotential (Eq. 1) and its nonlinear form (Eq. A1), plotted as function of the material volumetric-expansion rate and the state of charge. For silicon, the correction factor can be as large as 3.5. However, in the examples analyzed here, large compressive stresses limit Si lithiation to $20-30 \%$ of the theoretical capacity. Therefore, the correction factor only varies within the highlighted green region where it reaches at most the value of 1.25 .

where $\mathbf{P}$ is the first Piola-Kirchhoff stress tensor, ${ }^{j}$ and $\mathbf{F}^{e}$ is the recoverable (i.e., elastic) lattice distortion, and $t s C^{e}=F^{e T} F^{e}$. We have assumed a multiplicative decomposition of the total deformation gradient as $\mathbf{F}=\mathbf{F}^{e} \mathbf{F}^{a}$. The deformation that arises from Vegard's law is the anelastic deformation $\mathbf{F}^{a}=\mathbf{F}^{a}(c)$ and is function of the local concentration.

$$
\mathbf{P}=J \sigma \mathbf{F}^{-\mathbf{T}}
$$

We restrict the examples herein to the case of an isotropic volumetric expansion

$$
\mathbf{F}^{a}(c)=\left(1+\frac{\beta}{\rho_{h}} \mathbf{c}\right) \mathbf{I}
$$

where $\mathbf{I}$ is the identity matrix. By susbstituting the form of $\mathbf{F}^{a}$ (Eq. A3) in Eq. A1 we obtain

$$
\begin{aligned}
& \varpi^{M}\left(c, \mathbf{C}^{e}\right)=-\frac{\Omega}{3} \operatorname{Tr}\left(\mathbf{F}^{e T} \mathbf{P}\right)=-\frac{\beta}{\rho_{h}} \mathbf{P}: \mathbf{F}^{e}= \\
& =-\frac{\beta}{\rho_{h}} J \boldsymbol{\sigma} \mathbf{F}^{-T}: \mathbf{F}^{e}=-\frac{\beta}{\rho_{h}}\left(1+\frac{\beta}{\rho_{h}} \mathbf{c}\right)^{2} \operatorname{Tr} \boldsymbol{\sigma} \\
& \varpi^{M}(c, \boldsymbol{\sigma})=-\frac{\Omega}{3}\left(1+\frac{\Omega}{3} c\right)^{2} \operatorname{Tr} \boldsymbol{}
\end{aligned}
$$

The stress overpotential in Eq. A4 tends to the linear Larché and Cahn overpotential of Eq. 1 as the factor $(1+\Omega c / 3)^{2}=\left(1+\beta c / \rho_{h}\right)^{2}$ approaches the unity.

For intercalating compounds characterized by low partial molar volume (or, equivalently small Vegard's lattice misfit parameters), the diffusion potential is accurately represented by Eq. 1. The nonlinear model ${ }^{62}$ is suitable to capture the transport properties of materials with large chemical expansions such as silicon. Fig. 10 shows a contour plot of the values the correction factor $\left(1+\beta c / \rho_{h}\right)^{2}$ takes as a function of the characteristic volumetric expansion rate and the state of charge. For silicon, the correction factor can be as high as 3.5 , when $\mathrm{Si}$ is close to its fully lithiated state (relative concentration close to 1). However, in the analyses presented herein, Si state of charge remains limited to about $20-30 \%$ of its theoretical capacity. Within this range (green mark in Fig. 10) the correction factor does not raise above 1.25 and the two forms of diffusion potential produce equivalent results. 


\section{References}

1. Y. Qi, H. Guo, L. G. Hector, and A. Timmons, Threefold increase in the Young's modulus of graphite negative electrode during lithium intercalation, Journal of The Electrochemical Society, 157(5), A558 (2010).

2. L. Y. Beaulieu, K. W. Eberman, R. L. Turner, L. J. Krause, and J. R. Dahn, Colossa reversible volume changes in lithium alloys, Electrochemical and Solid-State Letters, 4(9), A137 (2001)

3. A. Timmons and J. R. Dahn, Isotropic volume expansion of particles of amorphous metallic alloys in composite negative electrodes for li-ion batteries, Journal of The Electrochemical Society, 154(5), A444 (2007).

4. Y. Qi and S. J. Harris, In situ observation of strains during lithiation of a graphite electrode, Journal of The Electrochemical Society 157(6), A741 (2010).

5. J. Christensen and J. Newman, Stress generation and fracture in lithium insertion materials, Journal of Solid State Electrochemistry 10(5), 293 (2006).

6. M. W. Verbrugge and Y. T. Cheng, Stress distribution within spherical particles undergoing electrochemical insertion and extraction., Electrochemical Society Trans actions, 16(13), 127 (2008)

7. W. H. Woodford, Y.-M. Chiang, and W. C. Carter, Electrochemical shock of intercalation electrodes: a fracture mechanics analysis, Journal of The Electrochemical Society, 157(10), A1052 (2010).

8. E. Markervich, G. Salitra, M. Levi, and D. Aurbach, Capacity fading of lithiated graphite electrodes studied by a combination of electroanalytical methods, raman spectroscopy and $\{$ SEM $\}$, Journal of Power Sources, 146, 146 (2005), selected paper pressented at the 12th International Meeting on Lithium Batteries12th International Meeting on Lithium Batteries.

9. G. Sikha, B. N. Popov, and R. E. White, Effect of porosity on the capacity fade of a lithium-ion battery: Theory, Journal of The Electrochemical Society 151(7), A1104 (2004)

10. S. P. Nadimpalli, V. A. Sethuraman, D. P. Abraham, A. F. Bower, and P. R. Guduru, Stress evolution in lithium-ion composite electrodes during electrochemical cycling and resulting internal pressures on the cell casing, Journal of The Electrochemical Society, 162(14), A2656 (2015).

11. N. Liu, A pomegranate-inspired nanoscale design for large-volume-change lithium battery anodes, Nat. Nanotechnol., 9, 187 (2014)

12. H. Wu, Engineering empty space between si nanoparticles for lithium-ion battery anodes, Nano. Lett., 12, 904 (2012).

13. S. Chen, Silicon core-hollow carbon shell nanocomposites with tunable buffer voids for high capacity anodes of lithium-ion batteries, Phys. Chem. Chem. Phys., 14, 12741 (2012).

14. J. Luo, Crumpled graphene-encapsulated si nanoparticles for lithium ion battery anodes, J. Phys. Chem. Lett., 3, 1824 (2012).

15. N. Liu, A yolk-shell design for stabilized and scalable li-ion battery alloy anodes, Nano. Lett., 12, 3315 (2012).

16. K. Takada, Progress and prospective of solid-state lithium batteries, Acta Materialia 61(3), 759 (2013)

17. J. Li, C. Ma, M. Chi, C. Liang, and N. J. Dudney, Solid electrolyte: The key for high-voltage lithium batteries, Advanced Energy Materials, 5(4), 1 (2015).

18. J. G. Kim, B. Son, S. Mukherjee, N. Schuppert, A. Bates, O. Kwon, M. J. Choi, H. Y. Chung, and S. Park, A review of lithium and non-lithium based solid state batteries, Journal of Power Sources, 282, 299 (2015).

19. V. Thangadurai, S. Narayanan, and D. Pinzaru, Garnet-type solid-state fast li ion conductors for li batteries: critical review, Chem. Soc. Rev., 43, 4714 (2014).

20. E. Quartarone and P. Mustarelli, Electrolytes for solid-state lithium rechargeable batteries: recent advances and perspectives, Chem. Soc. Rev., 40, 2525 (2011).

21. K. Takada, N. Ohta, and Y. Tateyama, Recent Progress in Interfacial Nanoarchitectonics in Solid-State Batteries, Journal of Inorganic and Organometallic Polymer and Materials, 205 (2014).

22. M. Tatsumisago, M. Nagao, and A. Hayashi, Recent development of sulfide solid electrolytes and interfacial modification for all-solid-state rechargeable lithium batteries, Journal of Asian Ceramic Societies, 1(1), 17 (2013).

23. A. Mukhopadhyay and B. W. Sheldon, Deformation and stress in electrode materials for Li-ion batteries, Progress in Materials Science, 63 (February), 58 (2014)

24. S. Kim, S. J. Choi, K. Zhao, H. Yang, G. Gobbi, S. Zhang, and J. Li, Electrochemically driven mechanical energy harvesting, Nature Communications, 7, 10146 EP (2016).

25. D. Fauteux and R. Koksbang, Rechargeable lithium battery anodes: alternatives to metallic lithium, Journal of Applied Electrochemistry, 23(1), 1 (1993).

26. T. K. Bhandakkar and H. Gao, Cohesive modeling of crack nucleation under diffusion induced stresses in a thin strip: Implications on the critical size for flaw tolerant battery electrodes, International Journal of Solids and Structures, 47(10), 1424 (2010).

27. A. F. Bower and P. R. Guduru, A simple finite element model of diffusion, finite deformation, plasticity and fracture in lithium ion insertion electrode materials, Modelling and Simulation in Materials Science and Engineering, 20 (4), 045004 (2012).

28. C.-W. Wang and A. M. Sastry, Mesoscale modeling of a li-ion polymer cell, Journal of The Electrochemical Society, 154(11), A1035 (2007)

29. S. Golmon, K. Maute, and M. L. Dunn, Numerical modeling of electrochemicalmechanical interactions in lithium polymer batteries, Computers \& Structures, 87(2324), 1567 (2009)

30. R. E. García,Y.-M. Chiang, W. Craig Carter, P. Limthongkul, and C. M. Bishop Microstructural modeling and design of rechargeable lithium-ion batteries, Journal of The Electrochemical Society, 152(1), A255 (2005).

31. M. Zhu, J. Park, and A. M. Sastry, Fracture analysis of the cathode in li-ion batteries: A simulation study, Journal of The Electrochemical Society, 159(4), A492 (2012).

32. R. T. Purkayastha and R. M. McMeeking, An integrated 2-d model of a lithium ion battery: the effect of material parameters and morphology on storage particle stress, Computational Mechanics, 50(2), 209 (2012).
33. C. Miehe, H. Dal, L.-M. Schänzel, and A. Raina, A phase-field model for chemomechanical induced fracture in lithium-ion battery electrode particles, International Journal for Numerical Methods in Engineering (2015).

34. K. Aifantis and J. Dempsey, Stable crack growth in nanostructured li-batteries, Journal of Power Sources, 143(12), 203 (2005).

35. I. Ryu, J. W. Choi, Y. Cui, and W. D. Nix, Size-dependent fracture of si nanowire battery anodes, Journal of the Mechanics and Physics of Solids, 59(9), 1717 (2011).

36. K. Aifantis and S. Hackney, Mechanical stability for nanostructured sn- and si-based anodes, Journal of Power Sources, 196(4), 2122 (2011).

37. S. Kalnaus, K. Rhodes, and C. Daniel, A study of lithium ion intercalation induced fracture of silicon particles used as anode material in li-ion battery, Journal of Power Sources, 196(19), 8116 (2011).

38. Y. Xia, T. Wierzbicki, E. Sahraei, and X. Zhang, Damage of cells and battery packs due to ground impact, Journal of Power Sources, 267, 78 (2014).

39. X. Zhang and T. Wierzbicki, Characterization of plasticity and fracture of shell casing of lithium-ion cylindrical battery, Journal of Power Sources, 280, 47 (2015).

40. F. Hao and D. Fang, Reducing diffusion-induced stresses of electrode-collector bilayer in lithium-ion battery by pre-strain, Journal of Power Sources, 242415 (2013).

41. H. B. Chew, B. Hou, X. Wang, and S. Xia, Cracking mechanisms in lithiated silicon thin film electrodes, International Journal of Solids and Structures, 51(23-24), 4176 (2014).

42. A. Drozdov, Constitutive equations for self-limiting lithiation of electrode nanoparticles in li-ion batteries, Mechanics Research Communications, 57, 67 (2014).

43. J. Ye, Y. An, T. Heo, M. Biener, R. Nikolic, M. Tang, H. Jiang, and Y. Wang, Enhanced lithiation and fracture behavior of silicon mesoscale pillars via atomic layer coatings and geometry design, Journal of Power Sources, 248, 447 (2014).

44. L. Greve and C. Fehrenbach, Mechanical testing and macro-mechanical finite element simulation of the deformation, fracture, and short circuit initiation of cylindrical lithium ion battery cells, Journal of Power Sources, 214, 377 (2012).

45. Y. Wang, Y. He, R. Xiao, H. Li, K. Aifantis, and X. Huang, Investigation of crack patterns and cyclic performance of ti-si nanocomposite thin film anodes for lithium ion batteries, Journal of Power Sources, 202, 236 (2012).

46. M. Pharr, Z. Suo, and J. J. Vlassak, Variation of stress with charging rate due to strainrate sensitivity of silicon electrodes of li-ion batteries, Journal of Power Sources, $\mathbf{2 7 0}$, 569 (2014)

47. J. K. Min, M. Stackpool, C. H. Shin, and C.-H. Lee, Cell safety analysis of a molten sodium-sulfur battery under failure mode from a fracture in the solid electrolyte, Journal of Power Sources, 293, 835 (2015).

48. C. Zhang, S. Santhanagopalan, M. A. Sprague, and A. A. Pesaran, Coupled mechanical-electrical-thermal modeling for short-circuit prediction in a lithium-ion cell under mechanical abuse, Journal of Power Sources, 290, 102 (2015).

49. Z. Ma, Z. Xie, Y. Wang, P. Zhang, Y. Pan, Y. Zhou, and C. Lu, Failure modes of hollow core-shell structural active materials during the lithiation-delithiation process, Journal of Power Sources, 290, 114 (2015).

50. C. Zhang, S. Santhanagopalan, M. A. Sprague, and A. A. Pesaran, A representativesandwich model for simultaneously coupled mechanical-electrical-thermal simulation of a lithium-ion cell under quasi-static indentation tests, Journal of Power Sources, 298, 309 (2015)

51. S. S. Damle, S. Pal, P. N. Kumta, and S. Maiti, Effect of silicon configurations on the mechanical integrity of silicon-carbon nanotube heterostructured anode for lithium ion battery: A computational study, Journal of Power Sources, 304, 373 (2016).

52. I. Laresgoiti, S. Kabitz, M. Ecker, and D. U. Sauer, Modeling mechanical degradation in lithium ion batteries during cycling: Solid electrolyte interphase fracture, Journal of Power Sources, 300, 112 (2015).

53. I. Ryu, S. W. Lee, H. Gao, Y. Cui, and W. D. Nix, Microscopic model for fracture of crystalline si nanopillars during lithiation, Journal of Power Sources, 255, 274 (2014).

54. Y. Dai, L. Cai, and R. E. White, Simulation and analysis of stress in a li-ion battery with a blended limn2o 4 and lini0.8co0.15al0.05o2 cathode, Journal of Power Sources, 247, 365 (2014)

55. H. Yang, F. Fan, W. Liang, X. Guo, T. Zhu, and S. Zhang, A chemo-mechanica model of lithiation in silicon, Journal of the Mechanics and Physics of Solids, $\mathbf{7 0}$ 349 (2014).

56. B. Dimitrijevic, K. Aifantis, and K. Hackl, The influence of particle size and spacing on the fragmentation of nanocomposite anodes for li batteries, Journal of Power Sources, 206, 343 (2012)

57. S. Lee, J. Yang, and W. Lu, Debonding at the interface between active particles and $\{\mathrm{PVDF}\}$ binder in li-ion batteries, Extreme Mechanics Letters, 6, 37 (2016).

58. J. Cannarella, C. Z. Leng, and C. B. Arnold, On the coupling between stress and voltage in lithium-ion pouch cells, SPIE Sensing Technology + Applications, 9115 91150K (2014).

59. F. Larché, J. W. Cahn, A linear theory of thermochemical equilibrium of solids under stress., Acta Metallurgica, 21, 1051 (1973).

60. R. W. Balluffi, S. M. Allen, and W. C. Carter, Kinetics of materials, John Wiley \& Sons, New Jersey, 2005.

61. F. Larche and J. Cahn, Thermochemical equilibrium of multiphase solids under stress, Acta Metallurgica, 26, 1579 (1978)

62. G. Bucci, Y.-M. Chiang, and W. Carter, Formulation of the coupled electrochemicalmechanical boundary-value problem, with applications to transport of multiple charged species, Acta Materialia, 62, 33 (2016). 
63. V. A. Sethuraman, M. J. Chon, M. Shimshak, V. Srinivasan, and P. R. Guduru, In situ measurements of stress evolution in silicon thin films during electrochemical lithiation and delithiation, Journal of Power Sources, 195(15), 5062 (2010).

64. D. M. Piper, T. a. Yersak, and S.-H. Lee, Effect of Compressive Stress on Electrochemical Performance of Silicon Anodes, Journal of the Electrochemical Society, 160(1), A77 (2012).

65. S.-B. Son, S. C. Kim, C. S. Kang, T. A. Yersak, Y.-C. Kim, C.-G. Lee, S.-H. Moon, J. S. Cho, J.-T. Moon, K. H. Oh, and S.-H. Lee, A highly reversible nano-si anode enabled by mechanical confinement in an electrochemically activated $\mathrm{Li}_{x} \mathrm{Ti}_{4} \mathrm{Ni}_{4} \mathrm{Si}_{7}$ matrix, Advanced Energy Materials, 2(10), 1226 (2012).

66. B. W. Sheldon, S. K. Soni, X. Xiao, and Y. Qi, Stress Contributions to Solution Thermodynamics in Li-Si Alloys, Electrochemical and Solid-State Letters, 15(1), A9 (2012)

67. J. Selsing, Internal stresses in ceramics, Journal of the American Ceramic Society, 44(8), 419 (1961)

68. J. D. Eshelby, The Determination of the Elastic Field of an Ellipsoidal Inclusion, and Related Problems, Proceedings of the Royal Society of London Series A241, 376 (1957).

69. A. Sakuda, A. Hayashi, and Y. Takigawa, Evaluation of elastic modulus of $\mathrm{Li}_{2} \mathrm{~S}-\mathrm{P}_{2} \mathrm{~S}_{5}$ glassy solid electrolyte by ultrasonic sound velocity measurement and compression test, Journal of the Ceramic Society of Japan, 121(11), 946 (2013).

70. A. Sakuda, A. Hayashi, and M. Tatsumisago, Sulfide Solid Electrolyte with Favorable Mechanical Property for All-Solid-State Lithium Battery, Scientific reports, 3, 2261 (2013).

71. J. E. Ni, E. D. Case, J. S. Sakamoto, E. Rangasamy, and J. B. Wolfenstine, Room temperature elastic moduli and Vickers hardness of hot-pressed LLZO cubic garnet, Journal of Materials Science 47, 7978 (2012).
72. Y.-H. Cho, J. Wolfenstine, E. Rangasamy, H. Kim, H. Choe, and J. Sakamoto, Mechanical properties of the solid Li-ion conducting electrolyte: $\mathrm{Li}_{0.33} \mathrm{La}_{0.57} \mathrm{TiO}_{3}$, Journal of Materials Science 47, 5970 (2012).

73. V. L. Chevrier and J. R. Dahn, First principles model of amorphous silicon lithiation, Journal of The Electrochemical Society, 156(6), A454 (2009).

74. L. Baggetto, R. A. H. Niessen, F. Roozeboom, and P. H. L. Notten, High energy density all-solid-state batteries: A challenging concept toward 3D integration, Advanced Functional Materials, 18(7), 1057 (2008).

75. V. a. Sethuraman, V. Srinivasan, and J. Newman, Analysis of Electrochemical Lithiation and Delithiation Kinetics in Silicon, Journal of the Electrochemical Society, 160(2), A394 (2013).

76. G. Bucci, S. P. Nadimpalli, V. A. Sethuraman, A. F. Bower, and P. R. Guduru, Measurement and modeling of the mechanical and electrochemical response of amorphous si thin film electrodes during cyclic lithiation, Journal of the Mechanics and Physics of Solids, 62, 276 (2014), sixtieth anniversary issue in honor of Professor Rodney Hill.

77. M. Kumari and N. Dass, Pressure-dependent self-diffusion and activation volume in solids: Sodium, Phys. Rev. B, 49, 844 (1994)

78. B. Yildiz, 'stretching' the energy landscape of oxides-effects on electrocatalysis and diffusion, MRS Bulletin, 39, 147 (2014).

79. W. H. Woodford, Electrochemical shock: Mechanical degradation of ion-intercalation materials, Ph.D. thesis, Massachusetts Institute of Technology (2013).

80. S. Li, R. A. Sauer, and G. Wang, The eshelby tensors in a finite spherical domainpart i: Theoretical formulations, Journal of Applied Mechanics, 74(4), 770 (2006).

81. J. D. Eshelby, The determination of the elastic field of an ellipsoidal inclusion, and related problems, Proceedings of the Royal Society of London A: Mathematical, Physical and Engineering Sciences, 241(1226), 376 (1957). 